\title{
Noise Estimation Technique for Multiple Copies Image Denoising
}

\author{
Somkait Udomhunsakul ${ }^{\mathrm{a}}$, Napa Sae-Bae ${ }^{\mathrm{b}}$ \\ ${ }^{a}$ Faculty of Engineering and Architecture \\ ${ }^{\mathrm{b}}$ Faculty of Science and Technology \\ Rajamangala University of Technology Suvarnabhumi \\ Nonthaburi, Thailand \\ *Corresponding Author: sudomhun@hotmail.com
}

\begin{abstract}
This paper proposed a noise estimation technique when multiple noisy image copies are available. In particular, the proposed technique estimated noise variance of one noisy image using the information of an original image from another copy of noisy images. Consequently, this proposed noise estimation could be used in conjunction with the state-of-the-art denoising algorithms to improve the quality of recovered images in terms of PSNR.

Experiments are performed on two widely-used image datasets in wide range of noise variances to confirm the accuracy of the proposed estimation technique. The result also reveals that, when the noise variance is small, the estimation derived from the traditional method is of high variation, depending on the image content. In this case, the proposed method achieves much better accuracy whereby resulting in a large improvement of recovered image quality. In addition, the results confirm that when they are two noisy image copies, denoising after fusing the images leads to the recovered image with better quality.
\end{abstract}

Keywords: noise estimation, image denoising, multiple noisy images.

\section{Introduction}

Image noise reduction has long been a classical problem that has clear implication in many image processing applications. Typically, image noise reduction techniques are typically focused on a single copy of noisy image. These techniques include, but are not limited to, low pass filter, e.g., moving average filters, median filters,
Gaussian filters, Wiener filters ${ }^{(1)}$, adaptive block-based SVD filters ${ }^{(2,3)}$ and wavelet thresholding methods ${ }^{(4,5,6)}$.

However, in some applications, a single image could be corrupted by noise for multiple times. Consequently, researchers have proposed noise reduction techniques that can utilize information from those multiple noisy copies of the same image source more effectively and resulting in the recovered image with better quality as compared to the traditional approaches. In 2000, S. Grace Chang et al have studied the impact of ordering between thresholding and fusing steps towards the enhancement of image quality when multiple copies of images are available ${ }^{(7)}$. More recently, Youssef et al have proposed the hierarchical multistage nonlinear filtering techniques to reduce noise on medical images ${ }^{(8)}$.

Complement to their work, this paper proposes a technique that can estimate noise variance more accurately than the traditional noise estimation technique. Specifically, the proposed technique utilizes information from another noisy image copy to search for the optimal noise variance based on the assumption that these image copies are originated from a single source and that they are corrupted by Additive white Gaussian noise (AWGN)(i.i.d) $)^{(9)}$. This proposed estimation technique could then be used in conjunction with the well-known image noise reduction algorithm, namely wavelet thresholding, to reconstruct the image with better quality as compare to the original approach.

The paper is organized as follows. In Section 2, the related background is described. Then, in Section 3, the proposed image denoising technique for multiple noisy image copies is explained in detail. Then, in Section 4, the 
definition of evaluation metrics that are used to assess the performance of the proposed denoising technique is described. Next, in Section 5, experimental setting and the results are presented. Finally, conclusion and future work is discussed in Section 6.

\section{Background}

\subsection{Additive White Gaussian Noise (AWGN)}

Generally, image noise can be divided into two types: additive and multiplicative noise. AWGN is an additive noise signal that each sample is drawn from Gaussian distribution with zero mean and variance $\sigma$. Specifically, the probability density function, denoted by $f(x)$, of Gaussian or normal distribution with zero mean and variance is defined as:

$$
f(x)=\frac{1}{\sqrt{2 \sigma^{2} \pi}} e^{-\frac{x^{2}}{2 \sigma^{2}}}
$$

\subsection{Wavelet denoising algorithm}

Image denoising techniques via wavelet thresholding is first proposed by Donoho and Johnstone in $1994^{(5)}$. Since then, there are many other proposals to improve the performance of image denoising algorithm based on this wavelet thresholding technique. In this paper, the wavelet thresholding method based on bivariate shrinkage functions, proposed by Selesnick et al., is adopted as an algorithm to reduce noise on a single noisy image ${ }^{(6)}$. The implementation used in this work can be found $a t^{(10)}$.

\section{The proposed image denoising technique}

In this section, the proposed image denoising method is described. Specifically, the proposed noise estimation technique is developed and used in conjunction with the well-known wavelet denoising algorithm. Alternately, image fusion could be performed either before or after denoising process in order to recover the final image. Details for each process are described as follows.

\subsection{Noise estimation}

In DWT denoising algorithm, the threshold is typically computed as a function of noise variance. Given a single noisy image copy, this noise variance is often estimated using median absolute deviation (MAD) technique $^{(5)}$. In this work, we argue that when multiple copies of noisy images become available, those noisy images can coordinately be used to estimate the level of noise variance more accurately than the traditional method, namely the MAD estimation. Specifically, noise variance can be search for by optimizing the mean square error (MSE) between the denoised image and another noisy image. For simplicity, this paper only considers the case when two copies of noisy images are available.

Let $I$ be the original image and $I_{1}$ and $I_{2}$ be the first and the second copy of noisy image corrupted by Additive white Gaussian noise (AWGN) (i.i.d) with noise variance $\sigma_{1}$ and $\sigma_{2}$ accordingly. Then, $I_{1}[i, j]$ and $I_{2}[i, j]$ can be formulated as:

$$
\begin{aligned}
& I_{1}[i, j]=I[i, j]+N\left(0, \sigma_{1}\right) \\
& I_{2}[i, j]=I[i, j]+N\left(0, \sigma_{2}\right)
\end{aligned}
$$

Let $\mathrm{I}_{1}{ }^{\prime}\left(\sigma_{1}{ }^{\prime}\right)$ be the denoised image derived from $I_{1}$ when noise variance is estimated at $\sigma_{1} \hat{\text {. The objective }}$ function is to search for the noise variance that minimizes the Mean Standard Error (MSE) between $\mathrm{I}_{1}{ }^{\prime}$ and $\mathrm{I}_{2}$. That is:

$$
\sigma^{(1)}=\operatorname{argmin}_{\sigma_{1}{ }^{\wedge}}\left\|\mathrm{I}_{2}-\mathrm{I}_{1}{ }^{\prime}\left(\sigma_{1}{ }^{\wedge}\right)\right\|_{2}
$$

Similarly, the noise variance of $\mathrm{I}_{2}$ can be estimated from:

$$
\sigma^{(2)}=\operatorname{argmin}_{\sigma_{2}{ }^{\wedge}}\left\|I_{1}-\mathrm{I}_{2}{ }^{\prime}\left(\sigma_{2}{ }^{\wedge}\right)\right\|_{2}
$$

where $\mathrm{I}_{2}{ }^{\prime}\left(\sigma_{2}{ }^{\wedge}\right)$ is the denoised image derived from $\mathrm{I}_{2}$ when noise variance is estimated at $\sigma_{2} \hat{\text {. }}$

\subsection{Image fusion technique}

When multiple noisy images are available, the recovered image is typically constructed by fusing those images using linear combination whether before or after denoising. If the fusion is performed before denoising, the fusion can be computed as the weighted average at pixel-wise level as follows.

Let $\sigma_{\mathrm{n}}$ be the noise variance for each copy of the noise image. Each pixel of the fused image is computed as:

$$
I_{f}[i, j]=\sum_{i=1}^{N}\left(w_{n} \times I_{i}[i, j]\right)
$$

where optimal weight, $\mathrm{w}_{\mathrm{n}}$, for each of the image copies is given by ${ }^{(7)}$ :

$$
w_{n}=\frac{1}{\sigma_{i}^{2}} \times \frac{1}{\sum_{i=1}^{N}\left(\frac{1}{\sigma_{i}^{2}}\right)}
$$

Then the fused image can be denoised using the following noise variance estimation: 


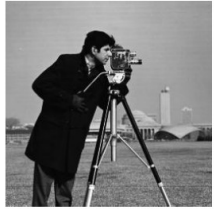

(a) Cameraman

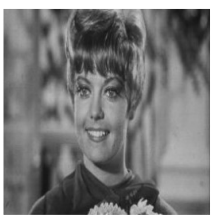

(h) Girl

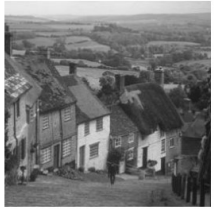

(b) Goldhill

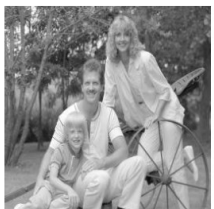

(i) Lena

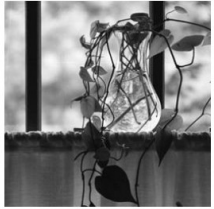

(c) Jug

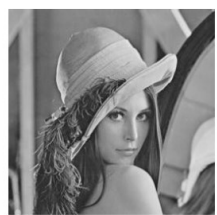

(j) Lenna

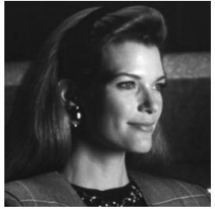

(d) Mam

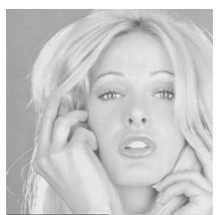

(k) Tiffany

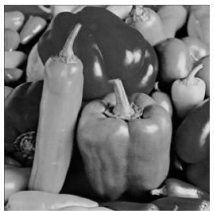

(e) Peppers

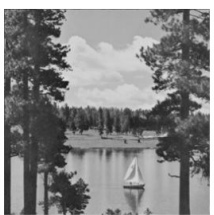

(1) Sailboat

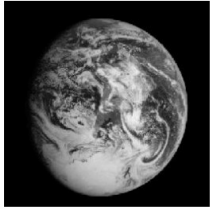

(f) Earth

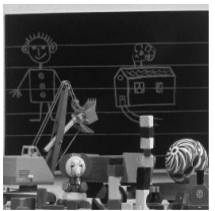

(m) Toys

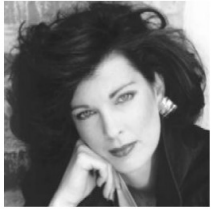

(g) Face

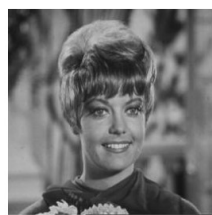

(n) Zelda

Fig.1.Original images in the in-house dataset.

$$
\sigma^{f}=\frac{1}{\sqrt{\sum_{i=1}^{N}\left(\frac{1}{\sigma_{i}^{2}}\right)}}
$$

\section{Evaluation metrics}

In this section, details for each evaluation metric that is used to assess the performance of the proposed image denoising algorithm are described. Specifically, one of the main contributions of this work is the noise variance estimation technique for multiple noisy image copies are available. Then this estimation technique is used in conjunction with the state-of-the-art image denoising algorithm. Therefore, the evaluation metric used in this paper aims to measure the accuracy of this estimation technique, and the quality of recovered images when noise variance is derived from the proposed estimation technique. Details for each metric are as follows.

\subsection{Mean Absolute Error (MAE)}

To assess the accuracy of noise estimation technique, the mean absolute error (MAE) is computed using the actual noise variance and the estimation derived from noisy images. The MAE can be computed acrossed all reference images as follows. Let $\sigma(\mathrm{i})$ and $\sigma^{\wedge}(\mathrm{i})$ be the true noise variance and the estimated noise variance of the $i^{\text {th }}$ image. The MAE is given by $^{(11)}$ :

$$
\operatorname{MAE}=\frac{\sum_{\mathrm{i}=1}^{\mathrm{N}}\left|\sigma(\mathrm{i})-\sigma^{\wedge}(\mathrm{i})\right|}{\mathrm{N}}
$$

where $\mathrm{N}$ is the number of images in the dataset.

\subsection{Peak Signal to Noise Ratio}

To assess the quality of recovered images, the recovered images are compared against an original image in terms of peak signal to noise ratio (PSNR). The PSNR can be computed as follows.

$$
P S N R=10 \log _{10} \frac{\left(2^{d}-1\right)^{2} W H}{\sum_{i=1}^{W} \sum_{j=1}^{H}\left(p[i, j]-p^{\prime}[i, j]\right)^{2}}
$$

where $\mathrm{d}$ is the number of bits that are used to represent the intensity of pixel. $\mathrm{W}$ and $\mathrm{H}$ are the width and height of the image. $p[i, j]$ and $p^{\prime}[i, j]$ are the intensity of the original image pixel and the recovered image pixel at the position $(i, j)$, respectively.

\section{Experimental results}

In this section, details about experiments to evaluate effectiveness the proposed denoising technique for images with multiple copies and the result are presented.

\subsection{Dataset}

Experiments are performed on two image datasets: the USC-SIPI Miscellaneous dataset and an in-house dataset. The first dataset comprises of 28 images; they are available at ${ }^{(12)}$. More details about this dataset can be found there. The second dataset comprises of 14 images that are widely used in image processing community. The images in this dataset are presented in Figure 1.

\subsection{Experiments}

The process to perform the experiment is as follows. First, for each image, multiple copies of noisy images are generated independently by corrupting the original images with additive Gaussian noise at the same noise variance starting from 5 to 10 . Then, the MAD noise estimation method is applied on each copy of noisy images to derive an estimated noise variance, namely, $\sigma_{\mathrm{MAD}}$. This estimation is also used to construct the search space for the proposed 
estimation technique. That is, the search space lies between $\widehat{\sigma}_{\mathrm{MAD}}-3$ to $\hat{\sigma}_{\mathrm{MAD}}+3$ where the interval is set at 0.25 . Finally, two techniques are used to recover the image from multiple copies of a noisy image. First, the denoised image using estimated noise variance from two different techniques is linearly combined. Secondly, the noisy images are linearly combined before denoising using the estimated noise variances derived from the proposed technique as discuss in the previous section.

\section{$5.3 \quad$ Results}

To demonstrate the accuracy of the proposed estimation technique, a noise estimation experiment is performed on the two datasets. Then, the noise estimation error using two estimation techniques are derived. The error distribution from of the USC-SIPI Miscellaneous image dataset when noise variance is set at 5 is presented in Figure 2. It is seen that, the estimation error of the proposed techniques is much smaller than the MAD technique. That is, at noise variance of 5 , the proposed technique estimates the value at 0.35 MAE, as compared to 1.51 MAE of the MAD one. For the in-house dataset, at the same noise level, the MAE of the proposed techniques is at 0.17 , as compared to 0.72 of the MAD one. Next, the same experiment is performed on both dataset when noise variance is varied from 5 to 10 in order to ensure the accuracy of the proposed estimation technique across wide range of noise level. The MAE result depicted in Figure 3 consistently confirms the accuracy at the proposed noise variance estimation technique.

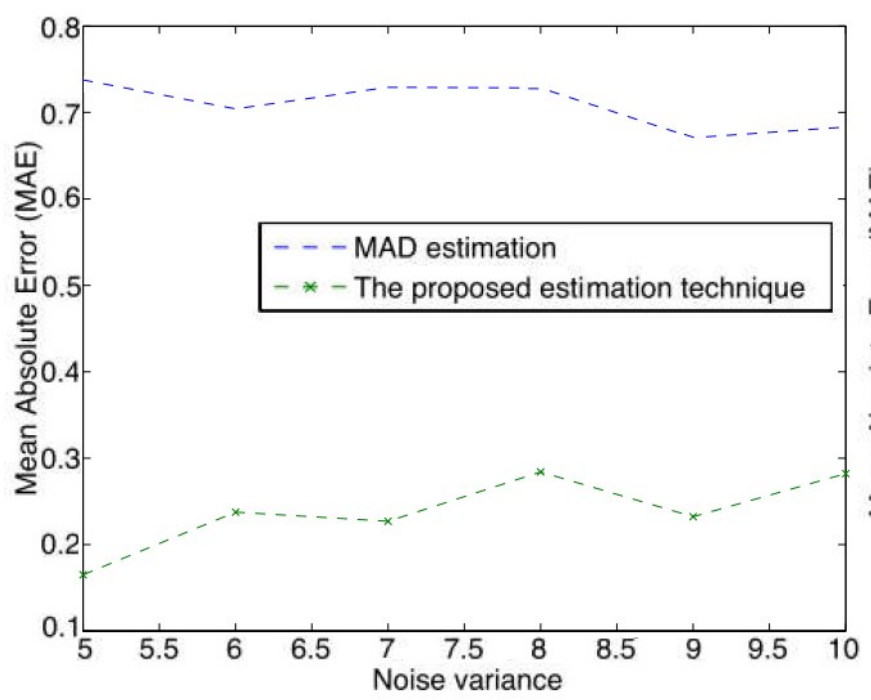

(a) The in-house dataset

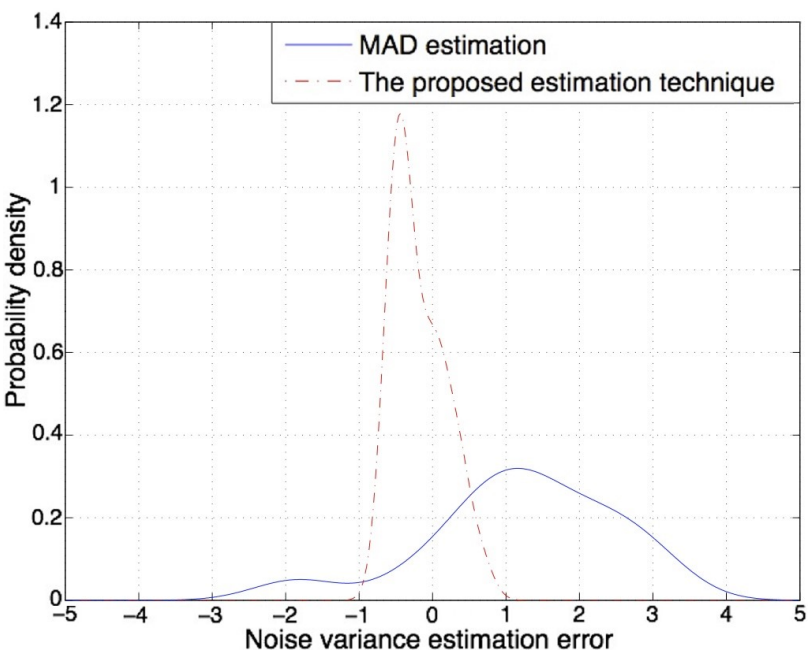

Fig. 2. The noise estimation error distribution derived from the USC-SIPI image dataset when noise variance is at 5.

This more accurate noise variance estimation can then be used in conjunction with the well-known noise reduction technique in order to enhance the quality of recovered image. To demonstrate the effect of more accurate estimation of noise variances towards quality enhancement of recovered images, the denoising experiment is performed using the state-of-the-art DWT denoising algorithm ${ }^{(6)}$. Specifically, three two-noisy-image-copy denoising approaches are compared. The first one, $\sigma_{\mathrm{MAD}}$, is to denoising each of the noisy image, using the wavelet threhsolding with noise variance estimated by the MAD technique and linearly combine them using the equation given in Eq.(6). The second one, $\sigma_{\text {Proposed, }}$ is similar to the first one except that the noise variance is estimated by the proposed method. The last one, $\sigma_{\mathrm{f}}$, is to first linearly combine the two image copies

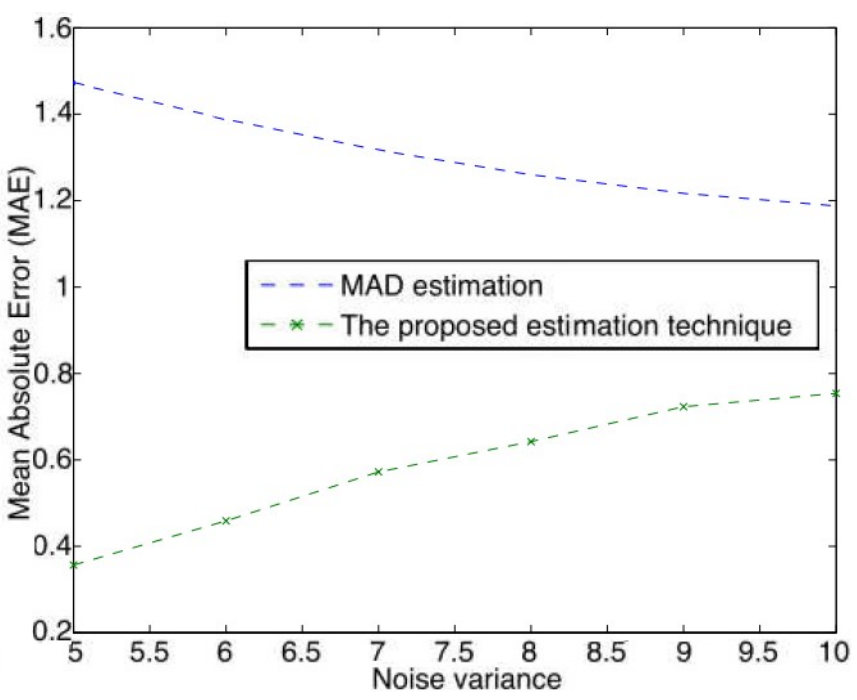

(b) The USC-SIPI dataset

Fig.3. Mean Absolute Error derived from the two noise estimation techniques at noise variances ranging from 5 to 10. 
using the equation given in Eq.(6) where the noise varianceis estimated using the proposed method. Then the fused image is denoised using the noise variance estimation given in Eg.(7). The result performed on the first dataset is reported in Table I. It clearly demonstrates the effectiveness of the proposed noise estimation technique as the quality of the recovered images are generally improved, as compared to the MAD estimation. In addition, it is also noticed that, in this setting, the quality of recovered images only gets better when image fusion is performed before denoising.

\subsection{Computational time}

The code used in all experiments is run on MATLAB R2014A with $2.4 \mathrm{GHz}$ Intel Core i5 CPU and 8 GB 1600 MHz DDR3 RAM Macbook machine with OS X 10.9.5. The average computational time of the proposed denoising algorithm for one image of size $256 \times 256$ is as followed. For the conventional DTW denoising algorithm, the proposed method using noise level estimated from each of the images and the denoised images are combined at last, and the proposed method using noise level estimated from combined noisy images, the execution time is at $0.05,0.64$, and 0.68 seconds, respectively.

Note that this Matlab execution code is performed in serialized fashion while it is also possible to perform the respective computation in parallel fashion which would make the computation time become linear to the conventional approach.

\section{Conclusion and future work}

In this work, the noise estimation technique for multiple noisy image copies is proposed. One application of this proposed technique is to be used in conjunction with other image noise reduction techniques to improve the quality of the recovered image in such scenario. The experiment performed on two datasets has consistently confirmed the accuracy of the proposed estimation technique and the image quality enhancement when it is applied to reduce additive noise on the image based on wavelet thresholding. However, this work only focuses on the simple case when only two noisy image copies are available. One area of the future work is to analyze the accuracy and confident level of the proposed estimation technique mathematically and to apply it to the application where more than two noisy image copies are available and where these images are not necessarily corrupted by the same noise variance.

\section{References}

(1) F. Jin, P. Fieguth, L. Winger, and E. Jernigan : "Adaptive wiener filtering of noisy images and image sequences", International Conference onImage Processing, Vol. 3, pp. 349-352, 2003.

(2) N. Sae-Bae and S. Udomhunsakul:“Adaptive block-based singular value decomposition filtering", Computer Graphics, Imaging and Visualisation, CGIV'07, pp. 298-303, 2007.

(3) N. Sae-Bae and S. Udomhunsakul: "Despeckling algorithm on ultra- sonic image using adaptive block-based singular value decomposition (invited paper)[6833-20]',Proceedings-SPIE The International Society for Optical Engineering, Vol. 6833, 2008.

(4) S. G. Chang, B. Yu, and M. Vetterli: "Adaptive wavelet thresholding for image denoising and compression", IEEE Transactions onImage Processing, Vol. 9, No. 9, pp. 1532-1546, 2000.

(5) D. L. Donoho and J. M. Johnstone : "Ideal spatial adaptation by wavelet shrinkage",Biometrika, Vol. 81, No. 3, pp. 425-455, 1994.

(6) I. W. Selesnick et al.,: "Bivariate shrinkage functions for wavelet-based denoising exploiting interscale dependency",IEEE Transactions onSignal Processing, Vol. 50, No. 11, pp. 2744-2756, 2002.

(7) S. G. Chang, B. Yu, and M. Vetterli: "Wavelet thresholding for multiple noisy image copies",IEEE Transactions on Image Processing, Vol. 9, No. 9, pp. 1631-1635, 2000.

(8) K. Youssef, N. Jarenwattananon, and L. Bouchard: "Feature-preserving noise removal", IEEE Transactions onMedical Imaging, Vol. 34, pp. 1822-1829, 2015.

(9) J. R. Hernandez, M. Amado, and F. Perez-Gonzalez: "DCT-domain watermarking techniques for still images: detector performance analysis and a new structure",IEEE Transactions onImage Processing, Vol. 9, No. 1, pp. 55-68, 2000.

(10) "Matlab implementation of wavelet transforms":http://eeweb.poly.edu/iselesni/WaveletSoft ware/index.html

(11)C. J. Willmott and K. Matsuura : "Advantages of the mean absolute error (mae) over the root mean square error (rmse) in assessing average model performance",Climate research, vol. 30, no. 1, pp. 79-82, 2005.

(12) "The usc-sipi image database": http://sipi.usc.edu/database/database.php 
Table 1. PSNR of the recovered images derived from three variations of the wavelet thresholding based image noise reduction technique when the images are corrupted with an additive white Gaussian noise at the variance of 5, 8, and 10 .

\begin{tabular}{|c|c|c|c|c|c|c|c|c|c|}
\hline \multirow{2}{*}{ Image name } & \multicolumn{3}{|c|}{$\sigma=5$} & \multicolumn{3}{|c|}{$\sigma=8$} & \multicolumn{3}{|c|}{$\sigma=10$} \\
\hline & $\hat{\sigma}_{M A D}$ & $\hat{\sigma}_{\text {Proposed }}$ & $\hat{\sigma}_{\text {Proposed }}^{f}$ & $\hat{\sigma}_{M A D}$ & $\hat{\sigma}_{\text {Proposed }}$ & $\hat{\sigma}_{\text {Proposed }}^{f}$ & $\hat{\sigma}_{M A D}$ & $\hat{\sigma}_{\text {Proposed }}$ & $\hat{\sigma}_{\text {Proposed }}^{f}$ \\
\hline Cameraman & 38.38 & 39.18 & 39.34 & 35.31 & 35.97 & 36.27 & 33.88 & 34.52 & $\mathbf{3 4 . 8 3}$ \\
\hline Goldhill & 37.25 & 38.29 & 38.52 & 34.44 & 35.08 & 35.41 & 33.10 & 33.70 & 34.04 \\
\hline Mam & 41.33 & 41.39 & 41.59 & 39.03 & 38.96 & 39.17 & 37.80 & 37.77 & 38.01 \\
\hline Peppers & 39.17 & 39.38 & 39.55 & 36.40 & 36.35 & 36.64 & 35.03 & 35.03 & 35.30 \\
\hline earth & 39.08 & 38.93 & 39.06 & 35.45 & 35.26 & 35.44 & 33.72 & 33.52 & 33.75 \\
\hline face & 41.04 & 40.96 & 41.28 & 38.32 & 38.27 & 38.49 & 37.13 & 36.96 & 37.32 \\
\hline girl & 39.28 & 39.72 & 39.83 & 37.12 & 37.21 & 37.43 & 35.98 & 36.02 & 36.29 \\
\hline lena & 36.77 & 38.10 & 38.32 & 33.76 & 34.68 & 34.93 & 32.34 & 33.13 & 33.47 \\
\hline lenna & 39.39 & 39.58 & 39.77 & 36.43 & 36.58 & 36.78 & 34.97 & 35.11 & 35.43 \\
\hline sailboat & 37.60 & 38.49 & 38.68 & 34.58 & 35.23 & 35.43 & 33.10 & 33.68 & 33.92 \\
\hline tiffany & 39.53 & 39.99 & 40.33 & 36.96 & 37.23 & 37.57 & 35.76 & 35.92 & 36.30 \\
\hline toys & 38.01 & 38.51 & 38.69 & 35.13 & 35.39 & 35.64 & 33.63 & 33.96 & 34.21 \\
\hline zelda & 40.23 & 40.39 & 40.61 & 37.62 & 37.62 & 37.94 & 36.39 & 36.35 & 36.66 \\
\hline
\end{tabular}

